\title{
Lorentz Microscopy of Magnetic Domain-Wall Pinning on Artificially Introduced Holes in Electrical Steel Sheets
}

\author{
Yoshikatsu Inada ${ }^{1, * 1}$, Zentaro Akase ${ }^{1, * 2}$, Daisuke Shindo ${ }^{1}$ and Akira Taniyama ${ }^{2}$ \\ ${ }^{1}$ Institute of Multidisciplinary Research for Advanced Materials, Tohoku University, Sendai 980-8577, Japan \\ ${ }^{2}$ Corporate Research and Development Laboratories, Sumitomo Metal Industries, Ltd., Amagasaki 660-0891, Japan
}

To investigate the interaction between magnetic domain walls and defects in an electrical steel sheet by transmission electron microscopy, size-controlled holes were artificially introduced into specimens by a focused ion beam. By applying an external magnetic field in a transmission electron microscope, the magnetic domain structures around the holes were dynamically observed by Lorentz microscopy. Similar magnetic domain structures were observed around the holes, but the domain-wall pinning strength was found to depend on the hole size. Micromagnetic analysis was performed to ascertain the magnetic domain structures around the holes and to investigate the change in the magnetic energy during domain-wall pinning. [doi:10.2320/matertrans.M2012099]

(Received March 14, 2012; Accepted April 20, 2012; Published June 6, 2012)

Keywords: magnetic domain-wall pinning, electrical steel sheet, in-situ observation, Lorentz microscopy

\section{Introduction}

Electrical steel sheets are a soft magnetic material that have a high permeability and a low coercive force. Consequently, they have been widely used in many devices including as magnetic cores in motors and transformers. To reduce the power consumption of such devices, it is important to reduce their energy loss in electrical steel sheets. One source of energy loss is the interaction between magnetic domain walls and defects. Transmission electron microscopy (TEM) can be used to observe both microstructures and magnetic domain structures at high magnifications. Using a recently developed magnetizing system for in-situ Lorentz microscopy with TEM, ${ }^{1,2)}$ it is now possible to observe the motion of a magnetic domain wall in a dynamic magnetic field. ${ }^{3)}$ Since the magnetic domain structure is sensitive to the size and crystallographic orientation of defects in TEM specimens, it is necessary to control these parameters in order to perform a systematic TEM investigation of the interactions between magnetic domain walls and defects such as precipitates.

In this study, size-controlled holes were artificially introduced into TEM specimens by a focused ion beam (FIB) in order to systematically investigate the interaction between a magnetic domain wall and holes. Surface-damagefree specimens were prepared using a scanning electron microscopy (SEM)/FIB multibeam system. The change in the magnetic domain structure as the magnetic domain wall passed across the hole was observed dynamically by Lorentz microscopy. Micromagnetic analysis was also performed to interpret the observation results and to assess the change in the magnetic energies during the interactions.

\section{Experimental Procedure}

TEM specimens were prepared from a doubly oriented electrical steel sheet, which has a $\{100\}\langle 001\rangle$ crystallographic texture and is highly anisotropic with two easy

\footnotetext{
${ }^{* 1}$ Graduate Student, Tohoku University

${ }^{* 2}$ Corresponding author, E-mail: akase@tagen.tohoku.ac.jp
}

(a)

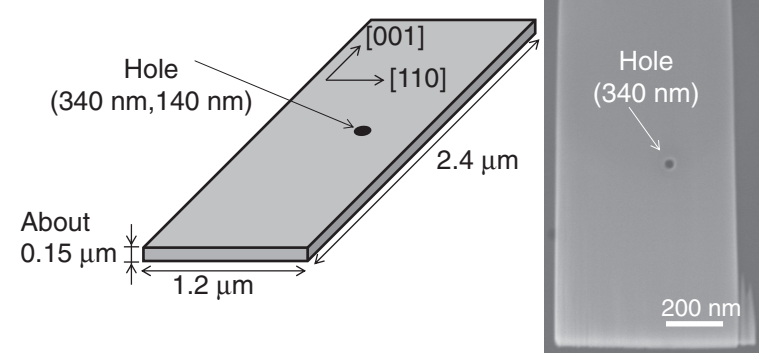

Fig. 1 Geometry and crystallographic orientation of specimen. (a) Schematic view and (b) SEM image of specimen.

magnetization axes in the sheet plane. ${ }^{4)}$ Figure 1(a) shows the shape and crystallographic orientation of a TEM specimen. Utilizing the FIB function of a JIB-4500 Multi Beam System, which has a gallium-ion gun for generating a FIB and an electron gun for SEM, small pieces were cut from the electrical steel sheet and they were fixed on collodionsupporting films of TEM microgrids. Each piece had dimensions of $1.2 \mu \mathrm{m} \times 6.0 \mu \mathrm{m} \times 0.15 \mu \mathrm{m}$ and the crystallographic orientations of the [001] and [110] directions lie along the long and short sides of the piece, respectively. The pieces contain no grain boundaries. Under these conditions, $180^{\circ}$ magnetic domain walls tend to appear near the center of the specimen. After attaching the pieces to the microgrids, each specimen was reinserted in the FIB system to produce size-controlled holes. The holes were formed using a gallium ion beam. The hole position was controlled by SEM observation. Note that the SEM observation did not damage the specimen surfaces. Two specimens with 140 and $340 \mathrm{~nm}$ diameter holes were prepared. Figure 1(b) shows a SEM image of the specimen with a $340 \mathrm{~nm}$ diameter hole.

Magnetic domain structures were observed by Lorentz microscopy using a JEM-3000F transmission electron microscope. A Lorentz lens and a magnetizing system were installed in this microscope. ${ }^{2,5,6)}$ In the magnetized state, a horizontal magnetic field is applied to a thin specimen by a 


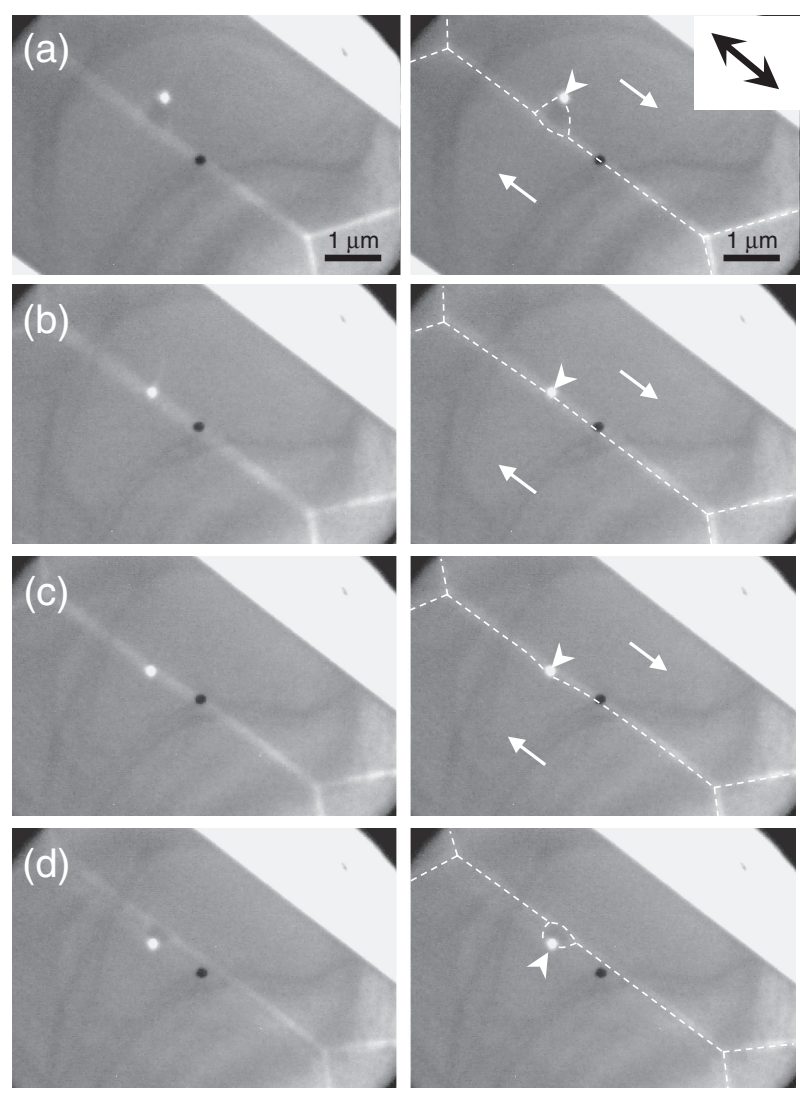

Fig. 2 Lorentz micrographs of specimen in an alternating magnetic field $(0.1 \mathrm{~Hz}, 4.3 \mathrm{kA} / \mathrm{m})$ captured by a video camera. The black dot at the center of each micrograph is a center spot of the fluorescent screen of the microscope, and thus the black dot is not related to the specimen. The dotted lines in the right column indicate the magnetic domain wall contrast and small arrows indicate the magnetization. The large arrow at the top right indicates the direction of the applied field and the arrowhead indicates a hole.

small electromagnet in the specimen holder. The domain-wall pinning process under an alternating magnetic field was observed using a video camera. To observe the magnetic domain structure in detail, Lorentz micrographs under a direct-current magnetic field were recorded by a slow-scan CCD camera.

A micromagnetic simulation was performed using the LLG Micromagnetics Simulator software ${ }^{7)}$ to investigate the magnetic domain structure and the magnetic energy. The crystallographic orientation of the model was the same as that of the specimen. The model had dimensions of $2.4 \mu \mathrm{m} \times$ $1.2 \mu \mathrm{m} \times 0.1 \mu \mathrm{m}$ and contained 60,100 and $200 \mathrm{~nm}$ square holes.

\section{Results and Discussion}

Figure 2 shows Lorentz micrographs of a specimen with a 140-nm-diameter hole under an alternating magnetic field (frequency: $0.1 \mathrm{~Hz}$; amplitude: $4.3 \mathrm{kA} / \mathrm{m}$ ). The dotted lines and arrow heads in the right column indicate the positions of a magnetic domain wall and a hole, respectively. The directions of the magnetization and the applied magnetic field are respectively indicated by the small arrows and the big arrow at the top right. This figure shows that the magnetic domain wall moves toward the top right and crosses the hole. (i)

(a)

(b)
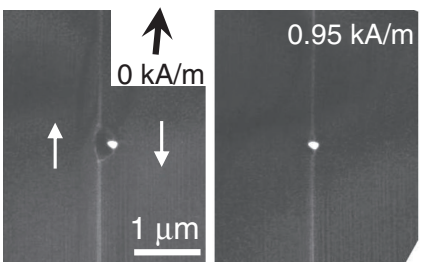

(iii)

)
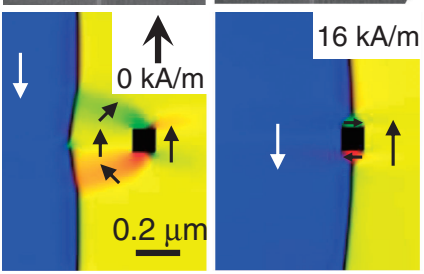

(c)
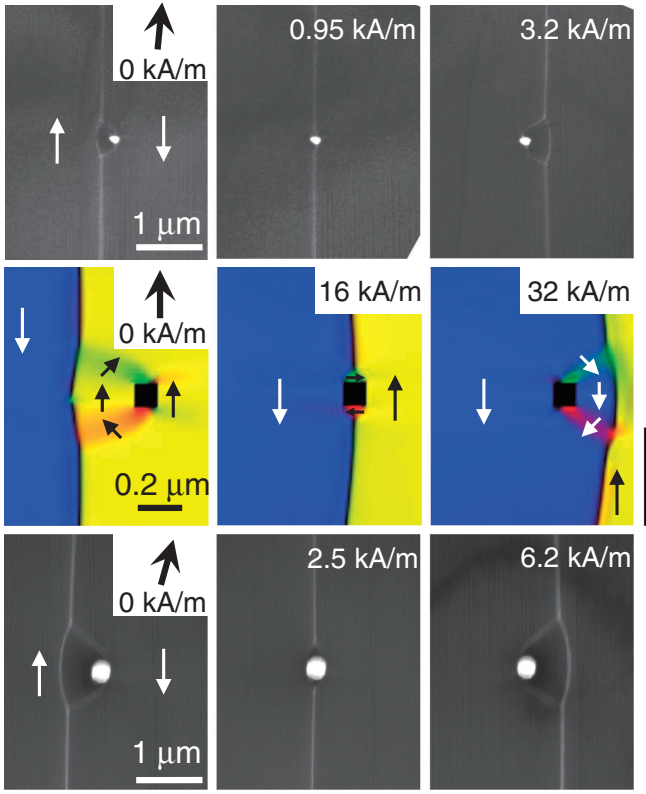

(d)
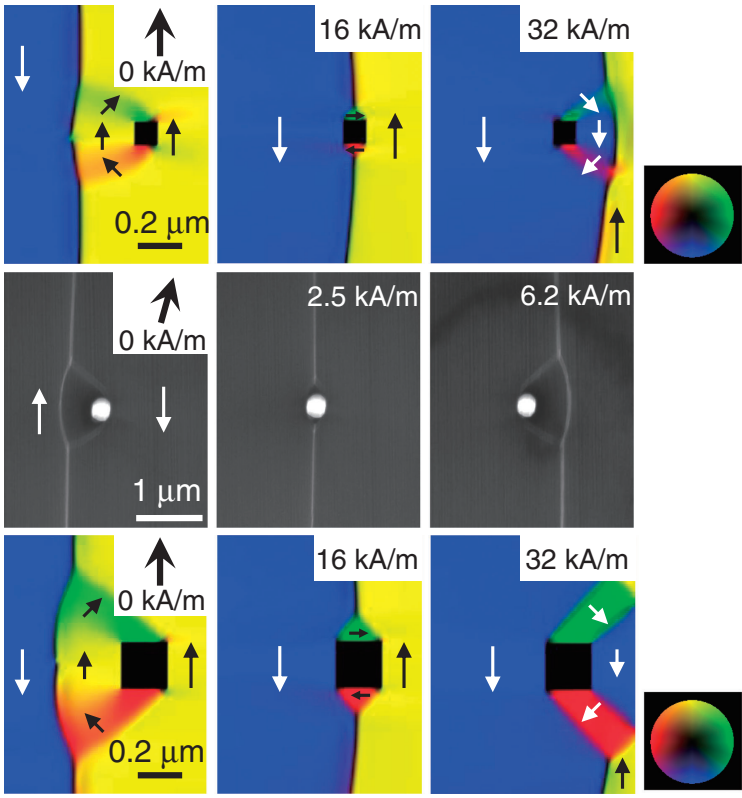

Fig. 3 Lorentz micrographs and simulations. Rows (a) and (c) show Lorentz micrographs with holes of size 140 and $340 \mathrm{~nm}$, respectively. Rows (b) and (d) show simulated magnetic domain structures for 100 and $200 \mathrm{~nm}$ square holes, respectively. The black square at the center of the figures is the hole. The small arrows indicate the magnetization directions. In rows (b) and (d), the magnetization directions are indicated by the color wheels on the right. The large arrows indicate the applied field directions and the numbers at the top right indicate the magnitude of the applied magnetic field.

The magnetic domain wall is pinned in Fig. 2(b) and it becomes curved around the hole in Fig. 2(c). The magnetic domain wall then leaves the hole (Fig. 2(d)).

To investigate the magnetic domain walls in detail, magnetic domain structures under a direct-current magnetic field were recorded by a slow-scan CCD camera. Figures 3(a) and 3(c) show Lorentz micrographs of specimens with 140 and $340 \mathrm{~nm}$ diameter holes under a directcurrent magnetic field, respectively. The direction of the applied field is indicated in the top right of column (i). When the external magnetic field was increased from 0 to $0.95 \mathrm{kA} / \mathrm{m}$, the magnetic domain wall was pinned at the hole; it left the hole when an external field of $2.7 \mathrm{kA} / \mathrm{m}$ was applied (Fig. 3(a)). Figure 3(c) shows a Lorentz micrograph of the specimen with a $340 \mathrm{~nm}$ diameter hole. The magnetic domain wall was pinned at the hole under an external magnetic field of $2.4 \mathrm{kA} / \mathrm{m}$ and it left the hole when an external magnetic field of $4.5 \mathrm{kA} / \mathrm{m}$ was applied. Although the magnetic domain structures in Figs. 3(a) and 3(c) have different sizes, they have similar morphologies. In both cases, the magnetic domain walls became curved to minimize the energy of the demagnetization field around the holes. Figures 3(b) and 3(d) show simulation results for 100 and $200 \mathrm{~nm}$ square holes, respectively. The arrows and color 
(a)

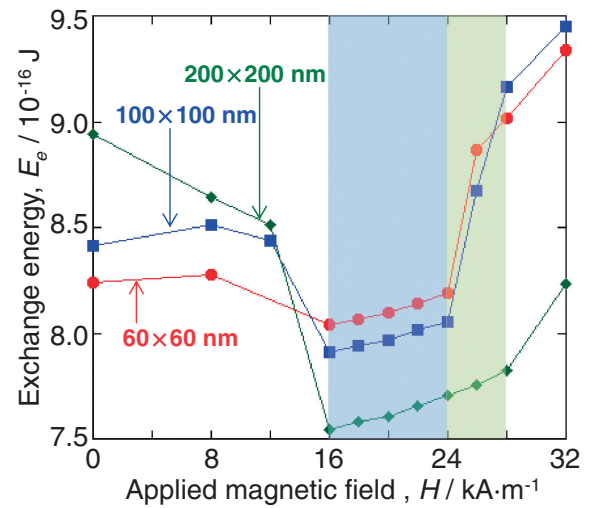

(b)

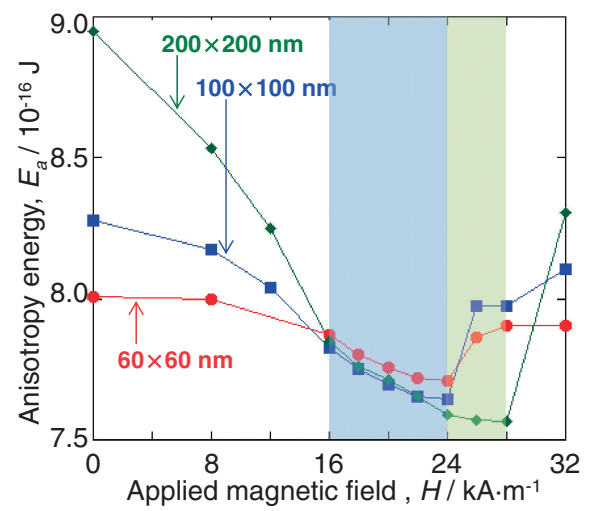

(c)

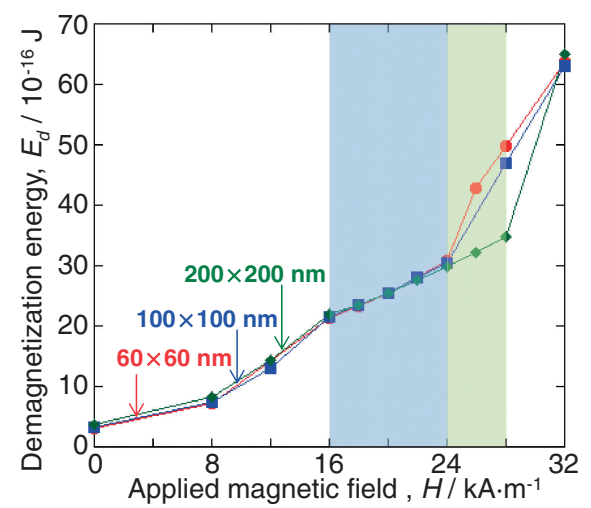

Fig. 4 Simulation results for specimens with different hole sizes: (a) exchange, (b) anisotropy and (c) demagnetization energies as functions of the applied magnetic field. The holes are $60 \times 60 \mathrm{~nm}(\mathrm{red}), 100 \times 100 \mathrm{~nm}$ (blue) and $200 \times 200 \mathrm{~nm}$ (green) squares.

wheels in these figures indicate the local magnetization directions. These figures clearly show that magnetic domains with opposite magnetization directions appear on either side of the holes in magnetic domain-wall pinning. These magnetic domain structures are considered to suppress the appearance of magnetic poles on the hole surfaces. In both cases, the simulated magnetic domain structures around the holes are consistent with the experimentally observed images. The morphologies of magnetic domain structures around different sized holes eventually become similar.

Here, we discuss the domain-wall pinning strength. Since the magnetic domain-wall motion was dynamically recorded, the external magnetic field range from the occurrence of domain-wall pinning to release of the domain wall could be evaluated for the two holes. The magnetic field ranges are 1.7 and $1.9 \mathrm{kA} / \mathrm{m}$ for holes with diameters of 140 and $340 \mathrm{~nm}$,
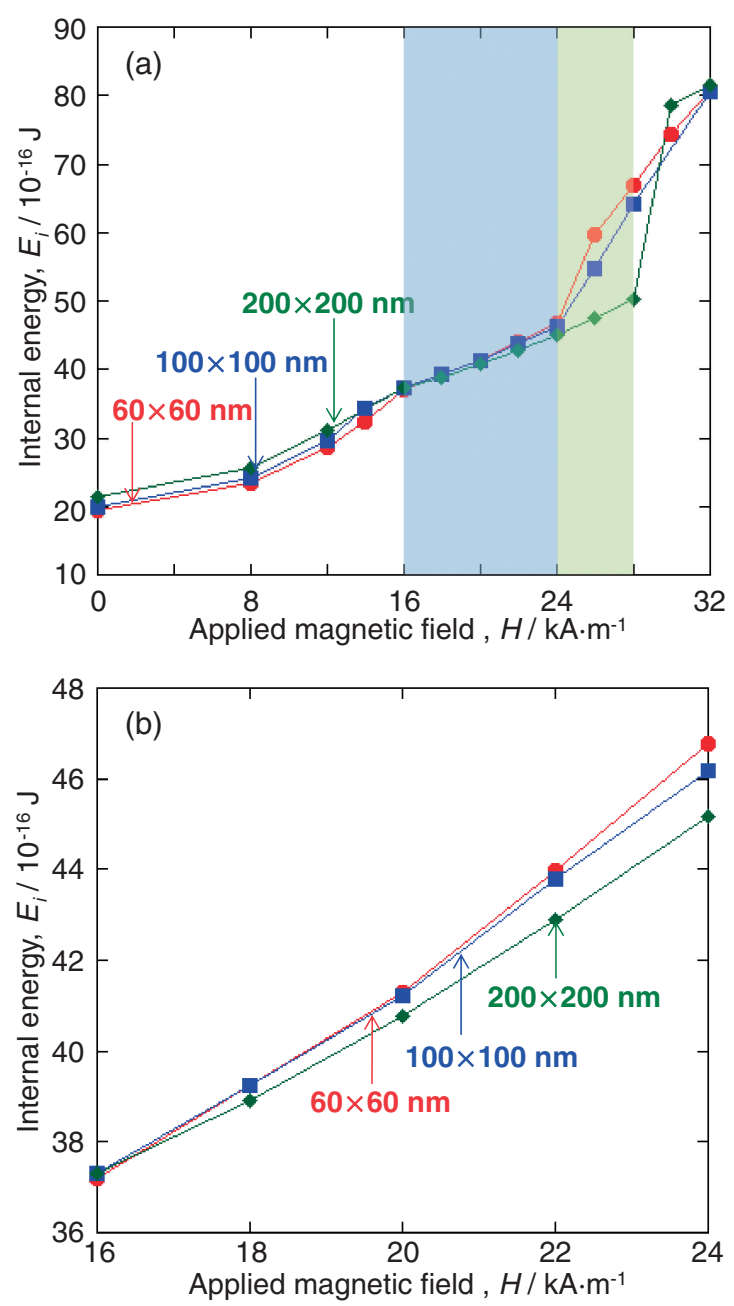

Fig. 5 (a) Simulated internal energy as a function of the applied magnetic field obtained from the data in Fig. 4. (b) Internal energy difference in the applied magnetic field range $16-24 \mathrm{kA} / \mathrm{m}$.

respectively. This difference indicates that the magnetic domain wall is pinned more strongly at the larger hole.

The magnetic energy, which consists of exchange, anisotropy, and demagnetization field energies, generated by the domain-wall pinning process is calculated by the LLG Micromagnetics Simulator. Figure 4 shows the relation between these energies as a function of the external magnetic field. The red, blue and green lines correspond to 60, 100 and $200 \mathrm{~nm}$ square holes, respectively. In all three cases, the magnetic domain walls are pinned in the blue region. On the other hand, in the green region, domain-wall pinning occurs only in the case of the $200 \mathrm{~nm}$ square hole. The calculation results reveal that when the magnetic domain wall is far from the hole, all three energies are larger than those for a bigger hole. In contrast, when the magnetic domain wall is pinned at the hole, the energy is smaller for the bigger hole. Figure 5(a) shows the relation between the applied magnetic field and the internal energy (i.e., the sum of the exchange, anisotropy, and demagnetization energies). Figure 5(b) shows the local internal energy in the blue region. In this region, the total internal energy decreases with increasing hole diameter during the domain-wall pinning process. This indicates that the magnetic domain wall is more stable at a bigger hole, which agrees with the experimental results. 


\section{Conclusion}

The interactions between the magnetic domain wall and artificially introduced holes in an electrical steel sheet were investigated by Lorentz microscopy. The findings are summarized below:

(1) Magnetic domain structures around different sized holes have similar shapes, whereas the domain-wall pinning strength is greater at bigger holes.

(2) The line shape of the magnetic domain wall and the magnetizing direction of the magnetic domains around the holes can be explained in terms of minimization of the demagnetization field and the suppression of the appearance of magnetic poles.

(3) Micromagnetic simulations clarified the detailed magnetization distribution around the holes and the changes in the magnetic energies during the pinning process are consistent with the Lorentz microscopy observations.

\section{Acknowledgment}

This work was supported by Grant-in-Aid for Young Scientists (B) (No. 22760528) from the Ministry of Education, Culture, Sports, Science and Technology (MEXT).

\section{REFERENCES}

1) M. Inoue, T. Tomita, M. Naruse, Z. Akase, Y. Murakami and D. Shindo: J. Electron Microsc. 54 (2005) 509-513.

2) Z. Akase and D. Shindo: J. Electron Microsc. 59 (2010) 207-213.

3) Z. Akase, D. Shindo, M. Inoue and A. Taniyama: Mater. Trans. 48 (2007) 2626-2630.

4) T. Tomida: Mater. Trans. 44 (2003) 1096-1105.

5) K. Shirota, A. Yonezawa, K. Shibatomi and T. Tanaka: J. Electron Microsc. 25 (1976) 303-304.

6) D. Shindo, Y.-G. Park, Y. Murakami, Y. Gao, H. Kanekiyo and S. Hirosawa: Scr. Mater. 48 (2003) 851-856

7) LLG micromagnetic simulator developed by Prof. M. Scheinfein, http:// llgmicro.home.mindspring.com. 